\title{
Susceptibility of Urinary Escherichia coli to Fosfomycin and Nitrofurantoin- Re-Exploration of Old and Forgotten Oral Antibiotics
}

\author{
Asha Pai K. B. ${ }^{1}$, Brinda Rajagopalan² \\ ${ }^{1}$ Department of Microbiology, KS Hedge Medical Academy, Mangalore, Karnataka, India. \\ ${ }^{2}$ KS Hedge Medical Academy, Mangalore, Karnataka, India.
}

\section{ABSTRACT}

\section{BACKGROUND}

Urinary tract infections (UTI) are one of the most common causes for patients to seek medical attention and also for prescription of antibiotics. Escherichia coli is the most common pathogen isolated from both complicated and uncomplicated UTI. Frequent and inappropriate use of antibiotics has led to antimicrobial resistance in the organisms. Multidrug resistance and carbapenem resistance are posing greater challenges to the clinicians. With only few antibiotics expected to be in the pipeline in the continuing five to ten years, the options that we are left with today are prudent use of antibiotics and re-exploration of old and forgotten antibiotics like nitrofurantoin, fosfomycin to help in combating the development of further resistance.

\section{METHODS}

This study was conducted in the Department of Microbiology, of our tertiary care hospital from March 2019 to June 2019. Retrospective analysis of results of urine culture was done. Isolates resistant to one antibiotic in at least three classes were considered as multidrug resistant. Susceptibility to fosfomycin and Nitrofurantoin among the MDR and non-MDR isolates were compared

\section{RESULTS}

Escherichia coli (66.67 \%) was the most common Enterobacteriaceae to be isolated, followed by Klebsiella pneumoniae (25.49\%). A very high susceptibility was noted to fosfomycin and Nitrofurantoin, with only $2.91 \%$ and $14.04 \%$ of the isolates being resistant to fosfomycin and nitrofurantoin respectively. Highest resistance was noted against ampicillin with 485 (83.05\%) being resistant followed by ciprofloxacin (65.75\%), norfloxacin (62.84\%), cotrimoxazole (47.09\%) and amoxicillin-clavulanic acid (36.99\%). About $49.14 \%$ of the isolates were found to be multi-drug resistant. There was no significant difference in the resistance rates to fosfomycin among the MDR (3.83 \%) and non-MDR isolates (2.02\%), while $20.55 \%$ of the MDR isolates and $7.74 \%$ of non-MDR isolates were resistant to nitrofurantoin.

\section{CONCLUSIONS}

Due to emergence of resistance to commonly used oral antibiotics in UTI, among the two older drugs- Nitrofurantoin and Fosfomycin, the latter can be used for treatment of acute cystitis, especially in cases due to MDR Escherichia coli, as Fosfomycin is equally effective against both MDR and non-MDR Escherichia coli isolated from urine samples while nitrofurantoin may be less effective against the MDR strains.

\section{KEY WORDS}

MDR, Escherichia coli, Nitrofurantoin, Fosfomycin
Corresponding Author:

Asha Pai K. B.,

Associate Professor,

Department of Microbiology,

KS Hedge Medical Academy,

Mangalore-575018, Karnataka, India.

E-mail: ashamkamath@gmail.com

DOI: $10.14260 /$ jemds/2020/200

Financial or Other Competing Interests: None.

How to Cite This Article:

Pai AKB, Rajagopalan B. Susceptibility of urinary Escherichia coli to fosfomycin and nitrofurantoin- re-exploration of old and forgotten oral antibiotics. J. Evolution Med. Dent. Sci. 2020;9(12):928-931, DOI: $10.14260 /$ jemds/2020/200

Submission 18-01-2020,

Peer Review 28-02-2020

Acceptance 05-03-2020,

Published 23-03-2020.

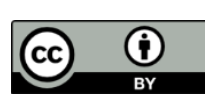




\section{BACKGROUND}

Urinary tract infections (UTI) are one of the most common causes for the patients to seek medical attention and also prescription of antibiotics. UTI occurring in otherwise healthy premenopausal women with normal anatomy and function of urinary tract are often termed as uncomplicated UTI. ${ }^{1}$ A complicated UTI is an infection which is usually associated with conditions such as obstruction (urinary calculi, prostatic hypertrophy), instrumentation, impaired voiding, metabolic abnormalities or immune- compromised states (renal transplant). ${ }^{2}$ Urinary tract infections can be caused by a wide variety of organisms. Escherichia coli is the most common pathogen isolated from both complicated and uncomplicated UTI. Other organisms include Klebsiella species, Proteus species, Staphylococcus saprophyticus, Enterococcus spp., Pseudomonas aeruginosa.1,3 Studies have shown that the organisms isolated from complicated UTIs tend to be more resistant to antibiotics. ${ }^{1}$ Frequent and inappropriate use of antibiotics has led to antimicrobial resistance in the organisms. ESBL producing Escherichia coli has been widely reported in the hospital set up and their frequency is increasing in the community strains as well. Multidrug resistance and carbapenem resistance are posing greater challenge to the clinicians. ${ }^{4}$

The ICMR, Antimicrobial Resistance Surveillance Network, 2018 data, reported that among the oral antibiotics used for treating UTI, very high resistance were recorded for levofloxacin (73.4\%), ciprofloxacin $(72.1 \%)$ and cotrimoxazole (60.6\%), while only $11.9 \%$ and $14 \%$ of the isolates were found to be resistant to two old antibiotics, Fosfomycin and Nitrofurantoin respectively. 5 The Treatment Guidelines for Antimicrobial Use in Common Syndromes (2019) by ICMR recommends nitrofurantoin or fosfomycin as drug of choice for empiric treatment of acute cystitis and cotrimoxazole, ertapenem or amikacin as alternative choice. However, it also recommends avoiding Fosfomycin and nitrofurantoin when there is suspicion of pyelonephritis or prostatitis/ presence of systemic features of infection. Piperacillin-tazobactam or Ertapenem are recommended as the drug of choice for the empiric treatment of acute pyelonephritis and complicated UTI's, while Imipenem or Meropenem or Amikacin may be used as alternatives. ${ }^{5}$

Antibiotic resistance among the isolates causing urinary tract infection varies widely depending on the geographical location, patient population, prior antibiotic use, patient ward/unit and other factors. With the current status of alarming development of antimicrobial resistance among bacteria causing urinary tract infection, knowledge of the spectrum and antibiotic susceptibility pattern of urinary isolates is essential not only for treatment but also to prevent the development of further resistance. With only few antibiotics expected to be in the pipeline in the continuing five to ten years, the options that we are left with today are prudent use of antibiotics and re-exploration of old and forgotten antibiotics like nitrofurantoin, fosfomycin to help combating the development of further resistance.

Hence this study was conducted to assess the antimicrobial susceptibility of Escherichia coli isolated from urine samples to oral antibiotics with special emphasis on resistance to nitrofurantoin and fosfomycin.

\section{METHODS}

This retrospective study was conducted in the Department of Microbiology, of our tertiary care hospital from March 2019 to June 2019. Ethical clearance was obtained from the institutional ethics committee. The study included the retrospective analysis of results of urine culture done in the Microbiology laboratory between January 2018 and December 2018. The urine samples received in the department were processed by standard Microbiology techniques. The isolates were identified by conventional biochemical reactions. The antibiotic susceptibility testing was carried out by automated system (Vitek 2 Compact). The isolates were classified as sensitive, intermediate and resistant to various antibiotics depending on the Minimum Inhibitory Concentration (MIC's) as per CLSI guidelines. Escherichia coli ATCC 25922 and Escherichia coli 35218 were used as controls. ${ }^{6}$

The data regarding the number of urine cultures, patient's age and gender, result of urine culture, identity of the organism isolated and their antibiotic susceptibility pattern to ampicillin, amoxicillin-clavulanic acid, ciprofloxacin, norfloxacin, cotrimoxazole, fosfomycin and nitrofurantoin were collected from the records maintained in the Department of Microbiology of our tertiary care hospital. All the data collected were entered in MS excel sheets and the antibiotic susceptibility pattern of Escherichia coli were analysed. Isolates resistant to at least three classes among Penicillins (ampicillin), beta lactam -beta lactamase inhibitor combination (amoxicillin-clavulanic acid), fluoroquinolones (ciprofloxacin, norfloxacin) and trimethoprim-sulphonamide combination (co-trimoxazole) were classified as multidrug resistant. Susceptibility to fosfomycin and Nitrofurantoin among the MDR and non-MDR isolates were compared.

\section{RESULTS}

Out of 5507 urine samples sent for culture and sensitivity testing to the Microbiology laboratory of or tertiary care hospital during Jan-Dec of 2018, 856 samples (15.54 \%) yielded significant growth of Enterobacteriaceae. Seven samples yielded growth of two organisms, hence a total of 863 isolates of Enterobacteriaceae were isolated from 856 samples. Out of the 856 positive samples, 457 and 399 were from female and male patients respectively. Escherichia coli (66.67 \%) was the most common Enterobacteriaceae to be isolated, followed by Klebsiella pneumoniae (25.49\%). The other species of Enterobacteriaceae isolated are shown in Table 1.

A very high susceptibility was noted to fosfomycin and Nitrofurantoin, with only $2.91 \%$ and $14.04 \%$ of the isolates being resistant to fosfomycin and Nitrofurantoin respectively. Of the 584 Escherichia coli isolates, highest resistance was noted against ampicillin with $485(83.05 \%)$ being resistant followed by Ciprofloxacin (65.75\%), Norfloxacin (62.84\%), 
Cotrimoxazole (47.09\%) and Amoxicillin-clavulanic acid (36.99\%). Further details are shown in Table 2.

Among the 584 Escherichia coli isolates 287 (49.14\%) isolates were found to be multi-drug resistant, of which 132 (45.99\%) were resistant to all four classes of antibiotics tested and $155(54.01 \%)$ to three classes of antibiotics. There was no significant difference in the resistance rates to fosfomycin among the MDR (3.83\%) and non-MDR isolates (2.02\%), while $20.55 \%$ of the MDR isolates and $7.74 \%$ of non-MDR isolates were resistant to Nitrofurantoin. The details are shown in table 3.

\begin{tabular}{|ccc|}
\hline Name of the Isolate & Number of Isolates & Percentage \\
Escherichia coli & 584 & 66.67 \\
Klebsiella pneumoniae & 220 & 25.49 \\
Klebsiella oxytoca & 25 & 2.9 \\
Enterobacter aerogenes & 16 & 1.85 \\
Enterobacter cloacae & 03 & 0.35 \\
Citrobacter koseri & 04 & 0.46 \\
Citrobacter freundii & 03 & 0.35 \\
Proteus mirabilis & 07 & 0.81 \\
Morganella morganii & 01 & 0.12 \\
Total & $\mathbf{8 6 3}$ & $\mathbf{1 0 0}$ \\
\hline \multicolumn{2}{c|}{ Table 1. Distribution of Different Species of Enterobacteriaceae } \\
\multicolumn{2}{c}{ Isolated from Urine Samples } \\
\hline
\end{tabular}

\begin{tabular}{|cccc|}
\hline Antibiotics & \multicolumn{3}{c|}{ Sensitive ( \%) Intermediate ( \%) Resistant ( \%) } \\
Ampicillin & $90(15.41)$ & $9(1.54)$ & $485(83.05)$ \\
Amoxicillin-clavulanic acid & $261(44.69)$ & $107(18.32)$ & $216(36.99)$ \\
Ciprofloxacin & $183(31.33)$ & $17(2.91)$ & $384(65.75)$ \\
Norfloxacin & $207(35.45)$ & $10(1.71)$ & $367(62.84)$ \\
Cotrimoxazole & $300(51.37)$ & $9(1.54)$ & $275(47.09)$ \\
Fosfomycin & $554(94.86)$ & $13(2.23)$ & $17(2.91)$ \\
Nitrofurantoin & $430(73.63)$ & $72(12.33)$ & $82(14.04)$ \\
\hline \multicolumn{4}{|c|}{ Table 2. Antibiotic Susceptibility Pattern of Escherichia coli } \\
\hline
\end{tabular}

\begin{tabular}{|ccccc|}
\hline & \multicolumn{2}{c}{ Fosfomycin } & \multicolumn{2}{c|}{ Nitrofurantoin } \\
& MDR & Non-MDR & MDR & Non-MDR \\
(n=287) & (N=297) & (n=287) & (N=297) \\
Sensitive (\%) & $268(93.38)$ & $286(96.30)$ & $174(60.62)$ & $256(86.20)$ \\
Intermediate (\%) & $8(2.79)$ & $5(1.68)$ & $54(18.81)$ & $18(6.06)$ \\
Resistant (\%) & $11(3.83)$ & $6(2.02)$ & $59(20.55)$ & $23(7.74)$ \\
\hline Table 3. Fosfomycin and Nitrofurantoin Susceptibility \\
of MDR and Non MDR Escherichia coli \\
\hline
\end{tabular}

\section{DISCUSSION}

Escherichia coli remains the most common organism to be isolated from urinary tract infections and accounts for two third of the urinary isolates. ${ }^{4}$ Irrational and widespread use of antibiotics has led to emergence of resistance due to production of penicillinases, cephalosporinases, Extended spectrum $\beta$-lactamases (ESBLs). Recently carbapenemase production has led to resistance to carbapenems such as imipenem, meropenem, ertapenem and Doripenem, which are considered as drug of choice for treating ESBL producing organisms. These organisms frequently tend to be multi-drug resistant which have a potential to spread widely and is a serious threat to the public health. ${ }^{7}$ With the alarming increase in drug resistance among bacterial pathogens and only few new antibiotics on the horizon, re-exploration of old and forgotten antibiotics like fosfomycin and nitrofurantoin are being considered as alternative approach for combating resistance.

Though fosfomycin was first isolated in 1969 in Spain and introduced throughout Europe in 1970s, the oral formulation, fosfomycin trometamol was introduced in 1995. It has a broad spectrum of activity and is highly effective against Escherichia coli. Variable susceptibilities have been ben reported in other urinary pathogens like Klebsiella spp.,
Proteus spp., Citrobacter spp., Enterobacter spp., Pseudomonas spp. and Enterococcus. Acinetobacter spp. and Morganella morganii are intrinsically resistant. 8 Nitrofurantoin was introduced into clinical practice in 1952. It is highly effective in the treatment of UTI caused by Escherichia coli, Citrobacter and Enterococcus. Its effectivity is less against Klebsiella and Enterobacter spp. ${ }^{7}$ Pseudomonas aeruginosa, Proteus spp., Morganella spp., Providencia spp., Serratia spp. are intrinsically resistant to nitrofurantoin.6,9 These two antibiotics have the advantage of attaining high concentrations in the urinary tract, minimal impact on gastrointestinal flora and a low propensity for resistance. ${ }^{8}$

In the present study, we analysed the in-vitro susceptibility of urinary Escherichia coli to commonly prescribed oral antibiotics like ampicillin, amoxicillinclavulanic acid, ciprofloxacin, norfloxacin and cotrimoxazole and two old antibiotics Nitrofurantoin and fosfomycin. We found that the isolates were highly resistant to ampicillin $(83.05 \%)$, followed by ciprofloxacin (65.75\%) Norfloxacin (62.84 \%), Cotrimoxazole (47.09 \%) and Amoxycillin/Clavulanate (36.99\%). This finding corelates with several other studies form India. Niranjan $\mathrm{V}$ et al reported that, Escherichia coli exhibited high levels of resistance to ampicillin (88.4\%), norfloxacin (74.2) and cotrimoxazole (64.2\%) very similar to our study. ${ }^{10}$ Similar observations were reported in yet another study by Kothari et al. with highest resistance to amoxicillin (82.3\%), followed by co-trimoxazole $(70 \%)$, ciprofloxacin $(64.2 \%)$ and Amoxycillin/Clavulanate $(58.4 \quad \%) .{ }^{11}$ A study from Puducherry, reported higher rates of resistance to Norfloxacin and cotrimoxazole of $77.6 \%$ and $73.4 \%$ respectively among urinary isolates of Escherichia coli. ${ }^{12}$ The result of yet another study from south India, were very much similar to our findings with high resistance noted against Amoxycillin/Clavulanate, norfloxacin, ciprofloxacin and cotrimoxazole. ${ }^{13}$ High resistance rates to these oral antibiotics may be due to irrational and uncontrolled use of these antibiotics over the past decade.

Our study revealed high susceptibility to fosfomycin with only $2.91 \%$ of the isolates being resistant. Similar observations were reported by Sabharwal et al., who found that $2.8 \%$ of Escherichia coli isolated from urine samples were resistant to fosfomycin. ${ }^{14}$ Very low resistance $(1.86 \%)$ to fosfomycin among urinary Escherichia coli was also reported by Banerjee S et al. from Eastern India, while a study from northern India found all the Escherichia coli isolates tested were susceptible to fosfomycin. ${ }^{15,16}$ Though resistance to fosfomycin develops rapidly in Escherichia coli in-vitro, resistance in clinical isolates is rare. This may be explained by the lowered fitness of these resistant organisms to establish in the urinary bladder. 17 Over $85 \%$ of our isolates were susceptible to Nitrofurantoin which correlates with several other studies from India and worldwide.10,11,18 The high susceptibility of Escherichia coli to nitrofurantoin may be due to limited use of the antibiotic because of its narrow spectrum of activity, limited indication (treatment of acute cystitis), narrow tissue distribution and limited contact with bacteria outside the urinary tract. 19

The occurrence of Multidrug resistance among urinary Escherichia coli is on a rise. UTI caused by multidrug-resistant (MDR) Escherichia coli not only increases the cost of treatment and morbidity but also mortality in severe cases. ${ }^{20}$ In our study $49.15 \%$ of the isolates were 
found to be multidrug resistant. A study conducted in a tertiary care hospital in North eastern Karnataka found $43 \%$ of their isolates to be multi drug resistant which correlates with the result of our study. ${ }^{20}$ Similar findings were also reported by other authors.20,21 Our study also found that the resistance to fosfomycin remains low even among the MDR isolates while resistance to Nitrofurantoin is more among MDR isolates when compared to non-MDR isolates. This corelates with the findings of the study by Gopichand et al who reported that fosfomycin retained the inhibitory effect on MDR isolates as well and Sultan A et al who reported that MDR Escherichia coli exhibited a decreased susceptibility to Nitrofurantoin. ${ }^{22,23}$

\section{CONCLUSIONS}

Due to emergence of resistance to commonly used oral antibiotics in UTI, among the two older drugs- Nitrofurantoin and Fosfomycin, the latter can be used for treatment of acute cystitis, especially in cases due to MDR Escherichia coli, as Fosfomycin is equally effective against both MDR and nonMDR Escherichia coli isolated from urine samples while Nitrofurantoin may be less effective against the MDR strains.

\section{REFERENCES}

[1] Mazzulli T. Diagnosis and management of simple and complicated Urinary Tract Infections (UTIs). Can J Urol 2012;19(Suppl 1):42-8.

[2] Nicolle LE, AMMI Canada Guidelines Committee. Complicated Urinary tract infections in adults. Can J Infect Dis Med Microbial 2005;16(6):349-60.

[3] Prakash D, Saxena RS. Distribution and antimicrobial susceptibility pattern of bacterial pathogens causing urinary tract infection in urban community of Meerut city, India. ISRN Microbiol 2013;2013:749629.

[4] Raja NS. Oral treatment options for patients with urinary tract infections caused by extended spectrum ßetalactamase (ESBL) producing Enterobacteriaceae. Journal of Infection and Public Health 2019;12(6):843-6.

[5] Treatment Guidelines for Antimicrobial use in common syndromes. $2^{\text {nd }}$ edn. New Delhi: ICMR 2019.

[6] CLSI. Performance Standards for Antimicrobial susceptibility testing. 29th edn. CLSI supplement M100. Wayne PA: Clinical and Laboratory Standards Institute, 2019.

[7] Eshetie S, Unakal C, Gelaw A, et al. Multidrug resistant and carbapenemase producing Enterobacteriaceae among patients with urinary tract infection at referral hospital, Northwest Ethiopia. Antimicrob Resist Infect Control 2015;4:12.

[8] Gardiner BJ, Stewardson AJ, Abbott IJ, et al. Nitrofurantoin and fosfomycin for resistant urinary tract infections: old drugs for emerging problems. Aust Prescr 2019;42(1):14-9.

[9] Cunha BA, Schoch PE, Hage JR. Nitrofurantoin: preferred empiric therapy for community-acquired lower urinary tract infections. Mayo Clin Proc 2011;86(12):1243-4.
[10] Niranjan V, Malini A. Antimicrobial resistance pattern in Escherichia coli causing urinary tract infection among inpatients. Indian J Med Res 2014;139(6):945-8.

[11] Khotari A, Sagar V. Antibiotic resistance in pathogens causing community - acquired urinary tract infections in India: a multicenter study. J Infect Developing Countries 2008;2(5):354-8.

[12] Srirangaraj S, Arunava K, Charles PMV, et al. Evaluation of in vitro susceptibility of fosfomycin among Enterobacteriaceae isolates from urine cultures: a study from Puducherry. J Lab Physci 2019;11(3):249-52.

[13] Sardar A, Basireddy SR, Navaz A, et al. Comparative evaluation of fosfomycin activity with other antimicrobial agents against E. coli Isolates from Urinary Tract Infections. J Clin Diagn Res 2017;11(2):DC26-DC9.

[14] Sabharwal ER, Sharma R. Fosfomycin: an alternative therapy for the treatment of UTI admist Escalating antimicrobial resistance. J Clin Diagn Res 2015;9(12):DC06-DC09.

[15] Banerjee S, Sengupta M, Sarker TK. Fosfomycin susceptibility among multidrug-resistant, extended-spectrum beta-lactamase-producing, carbapenem-resistant uropathogens. Indian J Urol 2017;33(2):149-54.

[16] Kumar D, Das A, Purbey MK, et al. Susceptibility of uropathogenic multidrug-resistant Escherichia coli to fosfomycin. J Acad Clin Microbiol 2017;19(2):101-4. http://www.jacmjournal.org/text.asp?2017/19/2/101/ 221507.

[17] Garau J. Other antimicrobials of interest in the era of extended-spectrum $\quad \beta$-lactamases: fosfomycin, nitrofurantoin and tigecycline. J Clin Microbiol Infect 2008;(14 Suppl 1):198-202.

[18] Lee DS, Lee SJ, Choe HS. Community-acquired urinary tract infection by Escherichia coli in the era of antibiotic resistance. Article ID 7656752. BioMed Research International 2018;2018:14.

[19] Mehta M, Bhardwaj S, Sharma J. Prevalence and antibiotic susceptibility pattern of multi-drug resistant Escherichia coli isolates from urinary tract infection (UTI) patients. International Journal of Life Sciences and Pharma Research 2012;2(4):L6-L11.

[20] Kulkarni SR, Peerapur BV, Sailesh KS. Isolation and antibiotic susceptibility pattern of Escherichia coli from urinary tract infections in a tertiary care hospital of North Eastern Karnataka. J Nat Sc Biol Med 2017;8(2):176-80.

[21] Das B, Mittal N, Goswami R, et al. Prevalence of multidrug resistance (MDR) and extended spectrum beta lactamases (ESBLs) among uropathogenic Escherichia coli isolates from female patients in a tertiary care hospital in North India. Int J Reprod Contracept Obstet Gynecol 2018;7(12):5031-6.

[22] Gopichand P, Agarwal G, Natarajan M, et al. In vitro effect of fosfomycin on multi-drug resistant gram-negative bacteria causing urinary tract infections. Infect Drug Resist 2019;12:2005-13

[23] Sultan A, Rizvi M, Khan F, et al. Increasing antimicrobial resistance among uropathogens: Is fosfomycin the answer? Urol Ann 2015;7(1):26-30. 